\title{
Abkürzungs- und Literaturverzeichnis
}

a. A.

a.a.O.

Abgaben-DV-LA

ABI.

Abs.

AcP

a. E.

a.F.

AG

AGBGB

AGGVG

\section{AktG}

Anh.

Anm.

AO

Arens

Arndt

Arnold-Meyer/Stolte

Art.

Aufl.

AVO-GBO

AV

Bad.Württ.LFGG

\section{Bärmann \\ Bärmann (WEG) \\ BAnz. \\ Bartholomeyczik \\ Bassenge}

anderer Ansicht

am angegebenen Ort

Durchführungsverordnung über Ausgleichsabgaben nach dem Lastenausgleichsgesetz

Amtsblatt des Regierungspräsidiums Saar (ab 1946, 49: der Verwaltungskommission des Saarlandes; ab 1947, 67: des Saarlandes), ab $1945 \mathrm{ff}$.

Absatz

Archiv für die civilistische Praxis (1.1818-149.1944, 150.1948/49ff.)

am Ende

alte Fassung

Amtsgericht

Ausführungsgesetz zum Bürgerlichen Gesetzbuch

Gesetz zur Ausführung des Gerichtsverfassungsgesetzes

Bayern: i.d.F. v. 7.11 .1974 (GVBl. S. 652)

Gesetz über Aktiengesellschaften und Kommanditgesellschaften auf Aktien (Aktiengesetz) vom 6.9.1965 (BGBl. I, 1089)

Anhang

Anmerkung

Abgabenordnung v. 16.3.1976 (BGBl. I, 613)

Willensmängel bei Parteihandlungen im Zivilprozeß 1968

Rechtspflegergesetz, Kommentar 1957.

Rechtspflegergesetz, Kommentar, 3. Aufl., 1978

Artikel

Auflage

Verordnung zur Ausführung der Grundbuchordnung

v. 8.8.1935 (RGBI.I, 1089)

Allgemeine Verfügung

Baden-Württembergisches Landesgesetz über die freiwillige Gerichtsbarkeit v. 12.2.1975 (GVBl. 116), geändert durch G. v. 14.12.1976 (GVBl. 618)

Freiwillige Gerichtsbarkeit und Notarrecht 1968

Wohnungseigentumsgesetz, Kommentar, 3. Aufl., 1975.

Bundesanzeiger

Erbrechts Kurzlehrbuch, 10. Aufl., 1975.

Kommentar zum FGG, 2. Aufl., 1976 


Baumbach-Duden
Baumbach-Hueck:
GmbHG
Baumbach-Hueck:
AktG
Baumbach-Lauterbach-
Albers-Hartmann
Baumgärtel
Baur
Baur
Bay.
BayBS

BayBSVJu.

Bay.JMBl.

BayNachlO

BayNachlG

BayObLG

BayObLGZ

\section{BB}

BBauG

Bd.

Bek.

Bem.

Betrieb

BeurkG

BGB

BGBI.

BGH

BGHSt.

BGHZ

BIGrdstBauW/R

BIGBW

Böhle-Stamschräder:

AnfG

Böhle-Stamschräder: KO

Böhle-Stamschräder: VglO
Handelsgesetzbuch mit Nebengesetzen ohne Seerecht

23. Aufl. 1978

Gesetz betreffend die Gesellschaften mit beschränkter

Haftung, Kurzkommentar, 13. Aufl. 1970

Aktiengesetz, Kurzkommentar, 13. Aufl. 1968

Zivilprozeßordnung, Kurzkommentar, 36. Aufl. 1978

Wesen und Begriff der Prozeßhandlung einer Partei im Zivilprozeß, 1957

Freiwillige Gerichtsbarkeit, Lehrbuch, 1955

Sachenrecht, Lehrbuch, 9. Aufl. 1977

Bayern, bayerisch

Bereinigte Sammlung des Bayerischen Landesrechts

1802-1956 BD. I-IV (Band und Seite)

Bereinigte Sammlung der bayerischen Justizverwaltungsvorschriften 1863 - 30.6.1957 Bd. I-VII

Bayerisches Justizministerialblatt (1947ff.)

Bayerische Nachlaßordnung v. 20.3.1903 (BayBSVJu. III, 166)

Bayerisches Nachlaßgesetz v. 9.8.1902 (BayBS III, 114)

Bayerisches Oberstes Landesgericht

Sammlung von Entscheidungen des Bayerischen Obersten

Landesgerichts in Zivilsachen (1.1901-34.1934/35)

N.F. 1. 1948-1951 ff.

Der Betriebsberater (Jahr u. Seite)

Bundesbaugesetz v. 23.6.1960 (BGBl. I, 341) i.d.F.

d. Bekanntmachung v. 18.8.1976 (BGBl. I, 2256)

Band

Bekanntmachung

Bemerkung

Der Betrieb (Jahr und Seite)

Beurkundungsgesetz v. 28. 8.1969 (BGBl. I, 1513)

Bürgerliches Gesetzbuch v. 18.8.1896 (RGBI. S. 195)

Bundesgesetzblatt (1950ff., ab 1951 zerfallend in Teil I und II)

Bundesgerichtshof

Entscheidungen des Bundesgerichtshofs in Strafsachen

(Band und Seite)

Entscheidungen des Bundesgerichtshofs in Zivilsachen

(Band und Seite)

Blätter für Grundstücks-, Bau- und Wohnungsrecht

(Jahr und Seite)

Blätter für Grundstücks-, Bau- u. Wohnungsrecht

(Jahr und Seite)

Anfechtungsgesetz, Kurzkommentar, 4. Aufl. 1965

Konkursordnung, Kurzkommentar, 12. Aufl. 1976

Vergleichsordnung, Kurzkommentar, 9. Aufl. 1977 
Abkürzungs- und Literaturverzeichnis

Boruttau-Klein

BRAO

Brox

Brügelmann

$\mathrm{BrZ}$

BT

Buchst.

Büro

BVerfG

BVerfGE

BVerfGG

BVersG

BVerwG

BWNotZ

\section{Daimer-Reithmann}

DDR

DFG

d.h.

Ditt

DJ

\section{DNotV \\ DNotZ \\ Dölle \\ DR}

DRZ

DRiZ

Dürkes

DVO

EG AO1977

EGBGB

EGGVG

Eickmann

Eickmann-Gurowski

Eickmann-Haegele-Riedel
Grunderwerbssteuergesetz, Kommentar 10. Aufl. 1977 und Ergänzungsband 1978

Bundesrechtsanwaltsordnung v. 1.8.1958 (BGBl. I, 565)

Erbrecht, Lehrbuch 1977

Bundesbaugesetz, Kommentar 1971

Britische Zone

Bundestag

Buchstabe

Das juristische Büro (Jahr und Seite)

Bundesverfassungsgericht

Entscheidungen des Bundesverfassungsgerichts (1.1952ff.)

Gesetz über das Bundesverfassungsgericht v. 12.3.1951

i.d.F. v. 3.2.1971 (BGBl. I, 105)

Bundesversorgungsgesetz i.d.F. v. 27.6.1977

(BGBl. I, 1037)

Bundesverwaltungsgericht

Mitteilungen aus der Praxis, Zeitschrift für das Notariat

in Baden-Württemberg (Jahr und Seite)

Die Prüfungs- u. Belehrungspflicht des Notars, 4. Aufl. 1974

Deutsche Demokratische Republik

Deutsche Freiwillige Gerichtsbarkeit (1.1936-9.1944)

das heißt

el Testament und Erbvertrag, 1972

Deutsche Justiz, Rechtspflege und Rechtspolitik, Amtl.

Organ des Reichsjustizministers (Jahr und Seite)

Zeitschrift des Deutschen Notarvereins (1.1901-33.1933,

Jahr und Seite; dann: DNotZ)

Deutsche Notar-Zeitschrift (33.1933-44.1944; Jahrg. 1950ff., Jahr und Seite)

Familienrecht, Lehrbuch 1964/1965.

Deutsches Recht (1.1931-15.1945; ab 9.1939

vereinigt mit Juristischer Wochenschrift)

Deutsche Rechtszeitschrift (1.1946-5.1950)

Deutsche Richterzeitung (Jahrg. 1950ff., Jahr und Seite)

Wertsicherungsklauseln 8. Aufl. 1972

Durchführungsverordnung

Einführungsgesetz zur Abgabenordnung v. 14.12.1976

(BGBl. I, 3341)

Einführung zum Bürgerlichen Gesetzbuch v. 18.8.1896

(RGBl. S.604)

Einführungsgesetz zum Gerichtsverfassungsgesetz

v. 27.1.1877 (RGBl. S.77)

Grundbuch-Verfahrensrecht, Lehrbuch, 1978

Grundbuchrecht, Lehrbuch, 1977

Rechtspflegergesetz, Kommentar 3. Aufl. $1970 \mathrm{ff}$. 
Abkürzungs- und Literaturverzeichnis

Einl.
Enneccerus
(und jeweilige Bearbeiter)
ErbbauVO
Erman
(und jeweiliger Bearbeiter)

FamRZ

ff.

FGG

Fn.

Gaentzsch
GBA
GBÄndVO
GBBerG
GBMaßnG
GBO
GBVereinfVO
GBVfg.

GenG

Gernhuber

GG

\section{Godin-Wilhelmi \\ GrdstVG}

Gruch
Grunsky
GS
Güthe-Triebel
GVBl.
GVG

Einleitung

Das Bürgerliche Recht, 15. Aufl., 1958/1959

Verordnung über das Erbbaurecht v. 15.1.1919 (RGBl. S. 72)

Das Bürgerliche Gesetzbuch, Handkommentar

6. Aufl. 1975 mit Nachtragsheft 1977

Ehe und Familie im privaten und öffentlichen Recht, Zeitschrift für das gesamte Familienrecht (Jahr und Seite) folgende (Seiten)

Reichsgesetz über die Angelegenheiten der freiwilligen

Gerichtsbarkeit v. 17.5.1898 (RGBl. S.771)

Fußnote

Städtebauförderungsgesetz, Kommentar, 1971, 2. Aufl. 1972

Grundbuchamt

Verordnung zur Änderung des Verfahrens in Grundbuchsachen v. 5.8.1935 (RGBl. I, 1065)

Gesetz über die Bereinigung der Grundbücher v. 18.7.1930 (RGBl. I, 305)

Gesetz über Maßnahmen auf dem Gebiet des Grundbuchwesens v. 20.12.1963 (BGBl. I, 986)

Grundbuchordnung i.d.F. der Bekanntmachung v. 5.8.1935 (RGBl. I, 1073)

Verordnung zur Vereinfachung des Grundbuchverfahrens v. 5.10.1942 (RGBl. I, 573)

Allgemeine Verfügung über die Einrichtung und Führung des Grundbuchs (Grundbuchverfügung) v. 8.8.1935 (RGBl. I, 637)

Gesetz betr. die Erwerbs- und Wirtschaftsgenossenschaften i.d.F. Bek. v. 20.5.1898 (RGBl. S. 810)

Familienrecht, Lehrbuch 2. Aufl. 1971

Grundgesetz für die Bundesrepublik Deutschland v. 23.5.1949 (BGBl. S. 1)

Aktiengesetz, Kommentar, 4. Aufl. 1971

Gesetz über Maßnahmen zur Verbesserung der Agrarstruktur und zur Sicherung land- und forstwirtschaftlicher

Betriebe (Grundstücksverkehrsgesetz)

v. 28.7.1961 (BGBl. I, 1091)

Beiträge z. Erläuterung d. Deutschen Rechts, begr. v.

Gruchot (Band und Seite)

Grundlagen des Verfahrensrechts, 1970

Gesetzessammlung

Grundbuchordnung, Kommentar, 6. Aufl. 1936/37

Gesetz- und Verordnungsblatt

Gerichtsverfassungsgesetz v. 27.1.1877 i.d.F. v.

9.5.1975 (BGBl. I, 1077) 
Abkürzungs- und Literaturverzeichnis

GVNW

Habscheid
Hachenburg
Haegele
Haegele

Halbs.

HannRpfl.

Hans

Heitzer-Oestreicher

Herbst

Hesse-Saage-Fischer

HessGVBI.

Heymann-Kötter
HGB
h.M.
HöfeOBrZ

Höfer-Huhn
Höfer-Huhn
Horber
HRR
Hueck
HW

Ingenstau

i.d.F.

i.S.

Jaeger
Jansen
Jansen

JBI.

JFG

JFGErg.

JM

JMBl.
Gesetz- und Verordnungsblatt für das Land Nordrhein-

Westfalen (1.1947 ff.)

Freiwillige Gerichtsbarkeit, Kurzlehrbuch, 6. Aufl. 1977

GmbH-Gesetz, Großkommentar, 6. Aufl. 1956/59

Beschränkungen im Grundstücksverkehr, 3. Aufl. 1970

Grundbuchrecht (Handbuch f. d. amtsgerichtiche Praxis)

5. Aufl. 1975

Halbsatz

Hannoversche Rechtspflege (1.1945-3. 1947; Jahr und

Seite; dann NdsRpfl.)

Städtebauförderungsgesetz, Kommentar, 1971

Bundesbaugesetz, Kommentar, 6. Aufl. 1977

Rechtspflegergesetz, Taschenkommentar, 2. Aufl., 1976

Grundbuchordnung, Kommentar, 4. Aufl. 1957

Gesetz- und Verordnungsblatt für das Land Hessen

(Jahr und Seite)

Handelsgesetzbuch, Kommentar, 21. Aufl. 1971

Handelsgesetzbuch v. 10.5.1897 (RGBl. S. 219)

herrschende Meinung

Höfeordnung Brit. Zone i.d.F. v. 26.7.1976

(BGBl. I, 1933)

Allgemeines Urkundenrecht, 1968

Beurkundungsgesetz, Kommentar, 1970

Grundbuchordnung, Kurzkommentar, 14. Aufl. 1977

Höchstrichterliche Rechtsprechung (Band und Seite)

Das Recht der Offenen Handelsgesellschaft, 4. Aufl. 1971

Haus und Wohnung (Jahr und Seite)

Erbbaurecht, Kommentar, 4. Aufl. 1972

in der Fassung

im Sinne

Konkursordnung, Großkommentar, 8. Aufl. $1958 \mathrm{ff}$.

Beurkundungsgesetz, Kommentar, 1971

Kommentar zum FGG, 2. Auflage, Bd. I 1969, Bd. II 1970, Bd. III 1971

Justizblatt

Jahrbuch der Entscheidungen in Angelegenheiten der freiwilligen Gerichtsbarkeit und des Grundbuchrechts (Band und Seite)

Entscheidungen des Kammergerichts (ab 15.1937) und des

Oberlandesgerichts München in Kosten-, Straf-,

Miet- und Pachtschutzsachen (1.1924-23.1943);

Band und Seite)

Justizministerium

Justizministerialblatt 


\section{JMBINRW}

JR

JuS

Justiz

JVBl.

JW

JWG

JZ

Justizministerialblatt für das Land Nordrhein-Westfalen (Jahr und Seite)

Juristische Rundschau (Jahr und Seite)

Juristische Schulung (Jahr und Seite)

Die Justiz, Amtsblatt des Justizministeriums Baden-Württemberg (Jahr und Seite)

Justizverwaltungsblatt (Jahr und Seite)

Juristische Wochenschrift (Jahr und Seite)

Gesetz für Jugendwohlfahrt i.d.F. d. Bek. v. 25.4.1977

(BGBl. I, 633, ber. 795)

Juristenzeitung (Jahr und Seite, Fortsetzung von DRZ und SJZ)

Erbschaftsteuergesetz, Kommentar, 4. Aufl. 1972

Handbuch der Erbengemeinschaft

Notar und Grundbuch, Bd. I 1971, Bd. II 1972

Freiwillige Gerichtsbarkeit, Kommentar

11. Aufl. 1978

Keidel-Stöber-Schmatz

KG

KGJ

\section{Kipp-Coing \\ Knaup-Ingenstau \\ KostÄndG}

KostO

\section{KostVfg.}

KRG

\section{LAG}

\section{Lange}

Lange-Wulff

Lent/Habscheid

Lent-Jauernig

Lent-Jauernig

Lent-Schwab

LG

LM

Registerrecht, 3. Aufl. 1976

Kammergericht (bei Entscheidungen nach 1945, soweit nichts anderes vermerkt, Kammergericht-West)

Jahrbuch für Entscheidungen des Kammergerichts in

Sachen der freiwilligen Gerichtsbarkeit pp. (1.1883-

53.1922; soweit nichts anderes angegeben, Abteilung A)

Erbrecht, Lehrbuch (Bd. 5 von Enneccerus-Kipp-Wolff)

12. Aufl. 1965

Bundesbaugesetz, Kommentar, 4. Aufl. 1969

Gesetz zur Änderung und Ergänzung kostenrechtlicher

Vorschriften v. 26.7.1957 (BGBl. I, 861)

Gesetz über die Kosten in Angelegenheiten der freiwilligen Gerichtsbarkeit (Kostenordnung) i.d.F. v.

26.7.1957 (BGBl. I, 960)

Kostenverfügung v. 28.2.1969

Kontrollratsgesetz

Gesetz über den Lastenausgleich (Lastenausgleichsgesetz) i. d. F. v. 1.10.1969 (BGBI. I, 1909)

Grundstücksverkehrsgesetz, Kommentar, 2. Aufl. 1964

Höfeordnung, 8. Aufl. 1968

Freiwillige Gerichtsbarkeit, Kurzlehrbuch, 4. Aufl.

Zivilprozeßrecht, Kurzlehrbuch, 18. Aufl. 1977

Zwangsvollstreckungs- und Konkursrecht, Kurzlehrbuch 14. Aufl. 1977

Sachenrecht, Kurzlehrbuch, 16. Aufl. 1977

Landgericht

Nachschlagewerk des Bundesgerichtshofs, herausgegeben von Lindenmaier, Möhring u. a. (1951 ff.) 
Abkürzungs- und Literaturverzeichnis

LwVG

MBl.

MDR

Mecke

Megow-Michel-Uhlich

Meikel-Imhof-Riedel

Meisner-Ring

Meisner-Stern-Hodes

\section{Mentzel-Kuhn \\ Meyer-Meulenbergh \\ MiZi}

MittBayNot.

MRG

MRVO

NdsMBI.

NdsRpfl.

n.F.

NJW

Nr.

\section{OGHBrZ \\ OGHZ \\ OLG \\ OLGRspr.}

\section{OLGZ}

Palandt

(und jeweiliger Bearbeiter)

Pikart-Henn

Planck

Pr.

Prölss-Martin

Prölss-Schmidt-Sasse

RBerG
Gesetz über das gerichtliche Verfahren in Landwirt-

schaftssachen v. 21.7.1953 (BGBl. I, 667)

Ministerialblatt

Monatsschrift für Deutsches Recht (Jahr und Seite)

Beurkundungsgesetz, Kommentar, 1970

Erbschaftsteuergesetz, Kommentar, 6. Aufl. 1974

Grundbuchrecht, Kommentar zur Grundbuchordnung,

6. Auflage, 1965/1969

Nachbarrecht in Bayern, 6. Aufl. 1972

Das Nachbarrecht im Bundesgebiet und West-Berlin mit

Ausnahme des Landes Bayern, 5. Aufl. 1970

Konkursordnung, Kommentar, 8. Aufl. 1976

Genossenschaftsgesetz, Kurzkommentar, 11. Aufl. 1970

Anordnung über Mitteilungen in Zivilsachen v. 1.10.1967

(bundeseinheitl.) s. z. B. BayJmB1. S. 127

Mitteilungen des Bayerischen Notarvereins, der Notarkasse und der Landesnotarkammer Bayern (Jahr und Seite)

Militärregierungsgesetz

Militärregierungsverordnung

Niedersächsisches Ministerialblatt (Jahr und Seite)

Niedersächsische Rechtspflege (Jahr und Seite)

neue Fassung

Neue Juristische Wochenschrift (Jahr und Seite)

Nummer

Oberster Gerichtshof für die Britische Zone

Entscheidungen des Obersten Gerichtshofs für die

Britische Zone in Zivilsachen (1.1949-4.1950)

Oberlandesgericht

Die Rechtsprechung der Oberlandesgerichte auf dem Gebiete

des Zivilrechts, herausgegeben von Mugdan und Falkmann (1.1900-46.1928)

Entscheidungen der Oberlandesgerichte in Zivilsachen einschließlich der freiwilligen Gerichtsbarkeit (Band und Seite)

Bürgerliches Gesetzbuch, Kurzkommentar, 38. Aufl. 1979

Freiwillige Gerichtsbarkeit, 1963

Bürgerliches Gesetzbuch, Kommentar, 5. Aufl. 1933

Preußisch

Versicherungsvertragsgesetz, Kurzkommentar, 21. Aufl. 1977

Versicherungsaufsichtsgesetz, Kurzkommentar, 8. Aufl. 1978

Gesetz zur Verhütung von Mißbräuchen auf dem Gebiete der Rechtsberatung v. 13.12.1935 (RGBl. I, 1478) 
Abkürzungs- und Literaturverzeichnis

\begin{tabular}{|c|c|}
\hline RdErl. & Runderlaß \\
\hline RdJ & Reichsminister der Justiz \\
\hline $\mathbf{R d L}$ & Recht der Landwirtschaft (Jahr und Seite) \\
\hline REGBrZ & $\begin{array}{l}\text { Rückerstattungsgesetz für die Britische Zone, Gesetz Nr.59, } \\
\text { v. 12.5.1949 (VOBl. BrZ S.152) }\end{array}$ \\
\hline Reithmann & Allgemeines Urkundenrecht, 1972 \\
\hline RG & Reichsgericht \\
\hline RGBl. & Reichsgesetzblatt (1871-1921; ab 1922-1945 Teil I und II) \\
\hline RGRK & $\begin{array}{l}\text { Das Bürgerliche Gesetzbuch, Reichsgerichtsrätekommentar, } \\
\text { 12. Aufl. } 1974 \mathrm{ff} \text {. }\end{array}$ \\
\hline RGZ & $\begin{array}{l}\text { Entscheidungen des Reichsgerichts in Zivilsachen } \\
\text { (Band und Seite) }\end{array}$ \\
\hline RHeimstG & Reichsheimstättengesetz v. 25.11.1937 (RGBI. I, 1291) \\
\hline $\begin{array}{l}\text { Ripfel } \\
\text { RJA }\end{array}$ & $\begin{array}{l}\text { Grundbuchrecht, } 1961 \\
\text { Entscheidungen in Angelegenheiten der freiwilligen Gerichts- } \\
\text { barkeit und des Grundbuchrechts, zusammengestellt } \\
\text { im Reichsjustizamt (Band und Seite) }\end{array}$ \\
\hline RMBl. & Reichsministerialblatt (Jahr und Seite) \\
\hline Rosenberg-Schwab & Zivilprozeßrecht, Lehrbuch, 12. Aufl. 1977 \\
\hline Rosenthal & $\begin{array}{l}\text { Das Bürgerliche Gesetzbuch, Kommentar, 15. Aufl. } 1965 \text { mit } \\
\text { Nachträgen 1966, 1968, } 1970\end{array}$ \\
\hline Rpfleger & Der Deutsche Rechtspfleger (Jahr und Seite) \\
\hline RpflG & Rechtspflegergesetz v. 5.11.1969 (BGBl. I, 2065) \\
\hline RSiedIG & Reichssiedlungsgesetz v. 11.8.1919 (RGBl. S.1429) \\
\hline S. & Sei \\
\hline$s$. & siehe \\
\hline Schlegelber & $\begin{array}{l}\text { Schlegelberger, Die Gesetze über die Angelegenheiten } \\
\text { der freiwilligen Gerichtsbarkeit für das Deutsche Reich } \\
\text { und Preußen, 7. Auflage, } 1956\end{array}$ \\
\hline SchlHA & Schleswig-Holsteinische Anzeigen (Jahr und Seite) \\
\hline $\begin{array}{l}\text { Schrödter } \\
\text { Schütz-Frohberg }\end{array}$ & $\begin{array}{l}\text { Bundesbaugesetz, Kommentar, 2. Aufl. } 1969 \\
\text { Bundesbaugesetz, Kommentar, 3. Aufl. } 1970\end{array}$ \\
\hline $\begin{array}{l}\text { Schutz-Fronberg } \\
\text { SeuffA }\end{array}$ & $\begin{array}{l}\text { Seufferts Archiv für Entscheidungen der obersten Gerichte } \\
\text { in den deutschen Staaten (Band u. Nr.) }\end{array}$ \\
\hline $\begin{array}{l}\text { SeuffBl. } \\
\text { Seybold-Hornig }\end{array}$ & $\begin{array}{l}\text { Seufferts Blätter für Rechtsanwendung (Jahr u. Seite) } \\
\text { Bundesnotarordnung, 5. Aufl. } 1976\end{array}$ \\
\hline Sichtermann & $\begin{array}{l}\text { Bedeutung und Behandlung der Eintragungen in Abt. II } \\
\text { des Grundbuches, 8. Aufl. } 1978\end{array}$ \\
\hline SJZ & $\begin{array}{l}\text { Süddeutsche Juristenzeitung (1. 1946-5.1950; Jahr und Seite; } \\
\text { dann: JZ) }\end{array}$ \\
\hline $\begin{array}{l}\text { Soergel } \\
\text { (und jeweiliger Bearbeiter) }\end{array}$ & $\begin{array}{l}\text { Das Bürgerliche Gesetzbuch, Kommentar, 11. Aufl. } \\
1978 \mathrm{ff} \text {. }\end{array}$ \\
\hline sog. & sogenannt \\
\hline $\begin{array}{l}\text { Staudinger } \\
\text { (und jeweiliger Bearbeiter) }\end{array}$ & $\begin{array}{l}\text { Das Bürgerliche Gesetzbuch, Kommentar, 11. Aufl. } \\
\text { ab 1954; 12. Aufl. ab } 1978\end{array}$ \\
\hline
\end{tabular}


Abkürzungs- und Literaturverzeichnis

StBauFG

\section{Stein-Jonas-Pohle \\ Steiner-Riedel \\ Steuer \\ st. Rspr.}

Thomas-Putzo

u.a.

usw.

Thieme

v.

vgl.

vo

VOBl.

Vorb.

Weitnauer-Wirths
WertpBG
Westermann
Wieczorek
Wieczorek
WiGBl.
WM
WohnSG
Wolff-Raiser
Wormit-Ehrenforth
z.B.
Zeller
Zöller
(und jeweiliger Bearbeiter)
ZPO
ZVG

ZZP

z.Zt.
Gesetz über städtebauliche Sanierungs- und Entwicklungsmaßnahmen in den Gemeinden (Städtebauförderungsgesetz) v. 27.7.71, BGBl. I, S.1125

Zivilprozeßordnung, Kommentar, 20. Aufl. ab 1977

ZVG, Kommentar, 8. Auflage, 1974

Flurbereinigungsgesetz, 2. Aufl. 1967

ständige Rechtsprechung

Zivilprozeßordnung, Kommentar, 10. Aufl. 1978

und andere

und so weiter

Grundbuchordnung, Kommentar, 4. Auflage, 1955

vom

vergleiche

Verordnung

Verordnungsblatt

Vorbemerkung

Wohnungseigentumsgesetz, Kommentar, 5. Aufl. 1974

Wertpapierbereinigungsgesetz v. 19.8.1949 (WiGBI. S. 295)

Sachenrecht, Lehrbuch, 6. Aufl. 1969

Zivilprozeßordnung, Kommentar, 2. Aufl. $1975 \mathrm{ff}$.

Zivilprozeßordnung (Handausgabe), 2. Aufl. 1966

Gesetzblatt der Verwaltung des Vereinigten Wirtschaftsgebietes

Wertpapier-Mitteilungen (Jahr und Seite)

Gesetz über die Aufschließung von Wohnsiedlungsgebieten v. 22.9.1933 (RGBl. I, 659)

Enneccerus-Kipp-Wolff, Lehrbuch des bürgerlichen Rechts, Band 3, 11. Auflage, 1964, Bearbeiter: Wolff und Raiser

Reichsheimstättengesetz, Kommentar, 4. Aufl. 1967

zum Beispiel

Zwangsversteigerungsgesetz, Kurzkommentar, 10. Aufl. 1978

Zivilprozeßordnung, Kommentar, 12. Aufl. 1978

m. Nachtrag 1971

Zivilprozeßordnung i.d.F. v. 12.9.1950 (BGBl. S. 533)

Gesetz über die $\mathrm{Zwangsversteigerung} \mathrm{und} \mathrm{die} \mathrm{Z}$ wangsverwaltung v. 24.3.1897 i.d.F. d. Bek. v. 20.5.1898 (RGBl. S.713)

Zeitschrift für Zivilprozeß (1.1879-63.1943; 64.

1950/51 ff.; Jahr und Seite)

zur Zeit 
